\title{
El Programa de Evaluación Docente en la UADY. Experiencia de la Implementación
}

\section{Teaching Assessment Program in the UADY. Implementation experience}

\author{
Norma Alicia Benois Muñoz*, Fabiola Isabel Gómez Cauich, \\ Maritza Minelli Briceño Caballero y Jessica Betzabé Zumárraga Ávila
}

Universidad Autónoma de Yucatán

La evaluación del desempeño docente tiene la finalidad de valorar la calidad de las responsabilidades en la enseñanza, aprendizaje y desarrollo del estudiante, sin embargo, es una tarea difícil de realizar (SEP, 2010). Debido a la necesidad de contar con un proceso de evaluación que contribuya con la mejora de la enseñanza, se creó el Programa de Evaluación Docente en la Universidad Autónoma de Yucatán (UADY). Éste propone promover la reflexión y el mejoramiento de la práctica docente, fortalecer los sistemas de formación y capacitación, así como estimular el interés de los profesores por el desarrollo profesional.

El objetivo de este trabajo es presentar la experiencia de su implementación y los resultados obtenidos en el primer ejercicio. Se realizó un estudio descriptivo con un enfoque mixto, predominantemente cuantitativo (Hesse-Biber, 2010). Participaron estudiantes y profesores del primer y segundo semestre de siete programas educativos. Los profesores obtuvieron resultados positivos en la evaluación de su desempeño, se reportó un avance entre los resultados del primer y segundo semestre y se observó correspondencia entre las respuestas otorgadas por estudiantes y profesores al evaluar el desempeño. De la misma forma, se identificaron las fortalezas y áreas de mejora del desempeño de los profesores.

Palabras clave: Evaluación docente, Educación superior, Estrategias de evaluación.

Teaching assessment is intended the purpose to make judgements regarding the quality of teachers' responsibilities accomplishment related to teaching, learning and students development, yet evaluating it seems to be a difficult task considering that teaching is very a complex and wide work (SEP, 2010). Because of the need of assessing teaching to better teachers' performance, the Autonomous University of Yucatan (UADY) developed "The Teaching Assessment Program" which promotes a reflexive practice of teacher's own performance. Through this program the UADY intend to strengthen the ongoing training system and encourage teachers into professional development.

The aim of this paper is to present the experience of their implementation and the results obtained in the first year. The current article presents a descriptive study with a mixed approach of investigation, quantitative predominantly (Hesse-Biber, 2010). This study presents the opinion of teachers and students of first and second semesters of seven different educational programs regarding their structure and the implementation as well as the teachers' performance. Although it was found that teachers usually obtain good assessment's results, their performance was better assessed in second semester than it was in the first one; both teachers and students coincided with teachers' performance assessment results. It also was found some strong areas and areas for improvement of the seven educational programs that in teachers and students' opinion must be considered to improve teachers' performance.

Keywords: Assessment of teachers, Higher education, Evaluation strategies.

\begin{tabular}{|c|c|c|}
\hline acorreo.u & Recibido: & 1 de marzo de 2016 \\
\hline 1980 & $1^{\text {a }}$ Evaluación: & 16 de marzo de 2016 \\
\hline ww & $2^{\text {a Evaluación: }}$ & 1 de abril de 2016 \\
\hline m.es & Aceptado: & 13 de abril de 2016 \\
\hline
\end{tabular}




\section{Introducción}

La evaluación docente es uno de los temas que ha cobrado más relevancia dentro del ámbito educativo, ya que se concibe como:

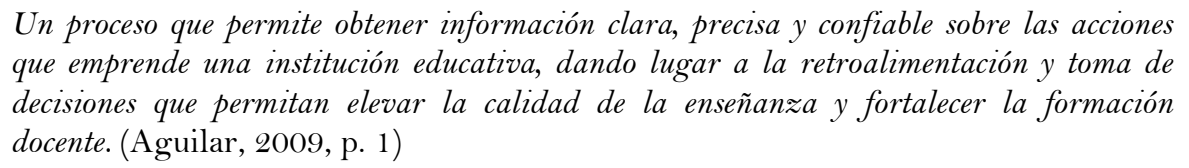

En sus inicios, la evaluación se centraba en la fiscalización, como un mecanismo de control; sin embargo, la tendencia afirma que debe enfocarse en la mejora de las actividades que se evalúan (García, Loredo, Luna y Rueda, 2008).

En concordancia con lo anterior, el Modelo Educativo para la Formación Integral (MEFI) de la Universidad Autónoma Yucatán (UADY) establece la evaluación al profesorado como un medio para promover su desempeño en términos del "perfil del profesor UADY” y considera que la evaluación debe hacerse desde diferentes perspectivas. A partir de esto, en el 2012 se elabora la propuesta para implementar el Programa de Evaluación Docente, cuyos propósitos son:

1. Promover la práctica reflexiva del docente sobre su propio desempeño y el compromiso frente a la calidad de la enseñanza.

2. Favorecer el mejoramiento de la práctica docente.

3. Contribuir al fortalecimiento de los sistemas de formación y capacitación del profesorado.

4. Estimular el interés de los profesores por el desarrollo profesional.

Para alcanzar tales propósitos, se toman en consideración tres elementos: evaluación por parte del estudiante, portafolio de evidencias y autoevaluación. Para ello, se diseñaron instrumentos con base en las actividades y competencias establecidas en el perfil del profesor UADY. El objetivo de este trabajo es presentar la experiencia de su implementación y los resultados obtenidos en el primer año de ejecución.

\section{Fundamentación teórica}

\subsection{El trabajo docente}

El trabajo docente es uno de los temas que ha generado gran interés de las instituciones, gobiernos y académicos de todo el mundo, ya que es reconocida la importancia que tiene el trabajo del profesor en la formación de estudiantes y la producción del conocimiento. Sin embargo, la complejidad de las tareas y los roles que desempeña, así como diversos factores que intervienen en su labor, plantea la necesidad de repensar cómo se concibe al profesor y la atención que debe ponerse en su formación y mejora continua.

En concordancia con esta tendencia, la UADY (2012, p. 48), declara en el Modelo Educativo vigente, que es necesario dimensionar el rol del profesor, para responder a las nuevas demandas que se presentan y establece el perfil deseable del profesor. En este se declara que el profesor es quien tiene la obligación de crear las condiciones de aprendizaje para el desarrollo de competencias; debe realizar actividades como facilitador, tutor, asesor, gestor y evaluador de los aprendizajes. Esto es:

a) Facilitador, porque planea las experiencias de aprendizaje significativo a partir del reconocimiento e identificación de los problemas del contexto; crea 
y recurre a escenarios de aprendizaje reales que favorezcan el desarrollo de las competencias dentro y fuera del aula, y apoya al estudiantado en la construcción del conocimiento.

b) Tutor, porque guía al estudiante en la toma de decisiones académicas, administrativas, personales y profesionales, favoreciendo la permanencia y conclusión exitosa de su formación profesional y, cuando es necesario, lo transfiere a otro tipo de apoyos.

c) Asesor, porque apoya al estudiante en relación con dificultades encontradas en el proceso de enseñanza y aprendizaje y orienta su buen desempeño académico en materia de contenidos y áreas disciplinares.

d) Gestor, porque procura el acceso a escenarios, recursos y contextos académicos a los que el estudiante por sí solo no podría acceder.

e) Evaluador, porque diseña la evaluación, propone criterios y evidencias de desempeño, emite juicios de valor en diversos momentos y circunstancias, $\mathrm{y}$ retroalimenta permanentemente el grado en que el estudiante ha desarrollado las competencias esperadas.

Del mismo modo, se declara que para el diseño de experiencias de aprendizaje, es necesario que el profesor considere las características del estudiantado y su contexto, el área disciplinar, los contenidos, los resultados de aprendizaje esperados, la modalidad de estudio (presencial, no presencial y mixta), la utilidad de las TIC, la disponibilidad de los recursos e infraestructura académica disponible y la posibilidad de acceder a escenarios de aprendizaje fuera del aula. Por tanto, en el marco de este Modelo Educativo, es necesario que los profesores se corresponsabilicen con la institución y esta, a su vez, favorezca el desarrollo de sus competencias que les permita ponerlo en práctica al interior y exterior de las aulas (UADY, 2012).

Como puede observarse, el Modelo Educativo vigente en la UADY otorga gran importancia al profesor, al considerarlo como uno de los actores principales del proceso de enseñanza y aprendizaje y como un participante activo en la formación del estudiante. La declaración de un perfil denota un interés de la institución en su trabajo docente, en su formación y actualización continua como la clave que garantice la calidad de la educación.

Por tanto, para el logro del perfil deseable del profesor es necesario promover la cultura de la evaluación y la promoción de la mejora continua entre el personal docente y en este sentido, la evaluación del desempeño docente surge como una acción institucional para favorecer lo anterior y los resultados obtenidos se consideran un insumo para que el profesor reflexione sobre su práctica.

\subsection{La evaluación del desempeño docente}

La evaluación del desempeño docente puede ser concebida de diversas formas. Las diferencias pueden estar determinadas por las políticas y el contexto de la institución en la que se lleva a cabo, los objetivos, uso de los resultados y los fines para los cuales fue creada. De acuerdo con Rizo (1999, p. 1), "la evaluación del desempeño docente es un proceso permanente, enmarcado dentro de una concepción de calidad de la educación y que se enfoca en el perfeccionamiento de la docencia en una institución educativa”. 
Por su parte, De Miguel (1998) la define como un proceso que permite al profesor detectar sus fortalezas y debilidades, así como los aspectos en los que debe mejorar. Afirma que en toda evaluación es necesario que el profesor se involucre y participe de forma activa para lograr la mejora a largo plazo.

En México, la Secretaría de Educación Pública define que la evaluación docente es:

Un proceso cuya finalidad es emitir juicios de valor sobre la calidad del cumplimiento de responsabilidades docentes en la enseñanza, aprendizaje y desarrollo de los estudiantes, a través de un seguimiento permanente que permita obtener información válida, objetiva y fiable para determinar los avances alcanzados en relación con los propósitos de la enseñanza y propósitos educativos. (SEP, 2010, p. 11)

Actualmente la mayoría de las Instituciones de Educación Superior han puesto en marcha iniciativas para evaluar el desempeño docente. En el contexto latinoamericano, se encuentra el Sistema de Evaluación del Desempeño Profesional Docente en Chile, el cual está orientado al fortalecimiento de la profesión docente. Desde su origen en la década de los noventa ha estado relacionado con diversos programas e iniciativas que buscan dignificar y restituir el carácter profesional de la actividad pedagógica (Bonifaz, 2011, p. 15).

En México, las acciones de evaluación del desempeño docente se identifican fundamentalmente como parte de los programas de compensación salarial, con el Programa de Mejoramiento del Profesorado (PROMEP), cuyo nombre cambió a partir del año 2014 a Programa para el Desarrollo Profesional Docente (PRODEP), el Programa de Fortalecimiento Institucional (PIFI) nombrado a partir de 2013 como Programa de Fortalecimiento de la Calidad de las Instituciones, entre otros. En la mayor parte de las Instituciones de Educación Superior, la evaluación docente es considerada como condición para el acceso a estos programas y también como insumo para la creación e implementación de iniciativas para el desarrollo profesional del profesor (Rueda, 2008).

Lo anterior denota la necesidad de crear más programas enfocados en la mejora y en la reflexión de la práctica del profesor. Para ahondar en este tema, se presentan los resultados de algunas investigaciones enfocadas en la evaluación del desempeño docente en las Instituciones de Educación Superior.

En primera instancia, Cisneros y Aguilar (2010) encontraron que la evaluación es necesaria para que los profesores obtengan retroalimentación de su desempeño docente y pueda proporcionar información valiosa para mejorar el mismo. Sin embargo, existen problemáticas relacionadas con la evaluación que deben tomarse en cuenta, una de ellas es la subjetividad, pues consideran que "la opinión de los estudiantes se ve afectada por su interés en el curso, la personalidad o estilo del profesor y especialmente por sus calificaciones" (Cisneros y Aguilar, 2010, p. 248).

Entre las dificultades identificadas en la investigación de Cisneros y Aguilar (2010) se encuentra el hecho de que, en la mayoría de los casos, no se evalúan todas las competencias de la práctica docente. Además, los profesores consideran que hay inconvenientes con los instrumentos utilizados, dado que algunos reactivos no son claros en su redacción, lo cual puede afectar la interpretación de los estudiantes al responder los cuestionarios. Aunado a lo anterior, se encontró que existe temor entre los docentes en cuanto al uso de los resultados y manifestaron su preocupación, ya que a nivel institucional se da importancia a los resultados para rendir cuentas respecto a indicadores propuestos por agencias externas, como el Padrón de Posgrados del 
Consejo Nacional de Ciencia y Tecnología (CONACYT) y los procesos de Acreditación de los Comités Interinstitucionales para la Evaluación de la Educación Superior (CIEES) (Cisneros y Aguilar, 2010).

De la misma forma, Rueda (2008) realizó un trabajo en donde se sintetizan las características más sobresalientes de la evaluación del desempeño docente en las universidades públicas de la Ciudad de México, entre sus conclusiones afirmó:

\begin{abstract}
Debido a la diversidad de ambientes institucionales, modelo educativo, infraestructura y condiciones del proceso de enseñanza aprendizaje, es importante contextualizar la evaluación es decir considerar en el diseño y la puesta en marcha el contexto institucional en que se encuentra. (Rueda, 2008, p. 10)
\end{abstract}

Por su parte, Cisneros y Aguilar (2010) enfatizaron la importancia de informar a los evaluados acerca del proceso además:

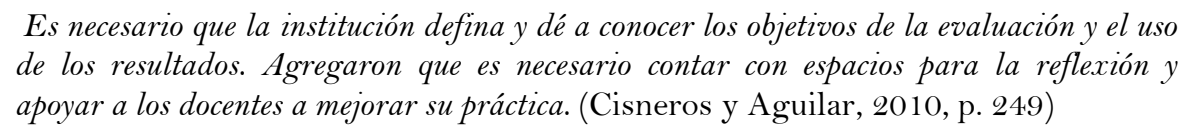

Los resultados del estudio de Cisneros y Aguilar (2010) indican que aunque comienza a considerarse el uso de otras fuentes de información en la evaluación de la docencia, tales como evaluación por pares, auto-evaluación y evaluación por supervisores o coordinadores, todavía no se utilizan en forma regular y no se han tomado en consideración las diferencias entre estas fuentes de información, así como sus fortalezas y debilidades cuando se usan para emitir un juicio sobre la calidad del desempeño docente.

Por su parte García, Loredo, Luna y Rueda (2008) mencionan que es necesario concebir la evaluación como un proceso tan complejo como la docencia misma. Asimismo, que la enseñanza puede ser juzgada de manera apropiada solo si se evalúa en el marco de los factores que la determinan. Bajo esta perspectiva se esperaría contar con sistemas acordes con el contexto educativo y características de los profesores.

Como puede observarse en las investigaciones presentadas existen múltiples formas de concebir la evaluación del desempeño docente, se han detectado problemáticas y aspectos a considerar dentro de los procesos de evaluación, por lo tanto, es recomendable que cualquier programa de evaluación docente que esté en proceso de construcción o en sus primeras etapas de implementación, considere esta información para contribuir al éxito y maximizar los beneficios del proceso.

Por tanto, el Programa de Evaluación Docente en la UADY surge con el propósito de contribuir al desarrollo y fortalecimiento de la profesión docente, específicamente al mejoramiento de la labor pedagógica de los profesores, con el fin de favorecer el aseguramiento de aprendizaje de calidad de los estudiantes y aportar información valiosa a los actores educativos. Para su construcción se tomó en consideración el contexto en el que se implementará, la opinión de los actores del proceso de enseñanza y aprendizaje, así como la congruencia con su principal referente: el Modelo Educativo vigente.

Este Programa concibe la evaluación con un carácter en primera instancia formativo y constituye una oportunidad para que el docente se conozca mejor desde el punto de vista profesional, identificando tanto sus fortalezas como los aspectos que puede mejorar. A continuación se detalla la estructura del Proceso. 


\subsection{Estructura del Proceso de Evaluación}

En la Universidad Autónoma de Yucatán, la evaluación del desempeño docente se realiza a través del Programa de Evaluación Docente que se ha implementado en los Programas Educativos de licenciatura, alineados al MEFI desde agosto de 2013. Este programa se integra por tres elementos:

a) Evaluación por parte del estudiante. Esta evaluación se realiza por medio de un formulario en versión electrónica que se aplica unas semanas previas al término del período escolar a evaluar.

b) Autoevaluación del profesor. Esta evaluación se realiza por medio de un formulario en versión electrónica alojada en una plataforma denominada "UADY Virtual", la cual se solicita al profesor que conteste en las últimas semanas del período escolar.

c) Portafolio de evidencias. Se solicita al profesor la creación de un portafolio electrónico el cual consta de tres partes: introducción, evidencias y reflexión final.

La ponderación de los elementos de evaluación se analizó y se determinó por un grupo de asesores expertos. Se sugirió dar mayor peso a la opinión de los estudiantes y dividir la ponderación de la evaluación por parte del propio profesor en dos elementos: el instrumento de autoevaluación y el portafolio de evidencias. La ponderación resultante fue la mostrada a la tabla 1.

Tabla 1. Ponderación de los elementos de la evaluación

\begin{tabular}{ll}
\hline ELEMENTO DE EVALUACIÓN & \\
\hline Evaluación por parte del estudiante & $60 \%$ \\
Autoevaluación del profesor & $15 \%$ \\
Portafolio de evidencias & $25 \%$ \\
\hline
\end{tabular}

Fuente: Elaboración propia.

Una vez recuperada la información de estos elementos, se obtiene una puntuación final sobre 100 puntos, los cuales permiten ubicar el nivel de desempeño del profesor de acuerdo a la tabla 2 .

Tabla 2. Nivel de desempeño de los profesores

\begin{tabular}{ll}
\hline RANGO & NIVEL DE DESEMPEÑO \\
\hline $91-100$ & Excelente \\
$76-91,99$ & Satisfactorio \\
$61-75,99$ & Suficiente \\
$1-60,99$ & Necesita mejorar \\
\hline Fuente: Elaboración
\end{tabular}

Fuente: Elaboración propia.

En virtud de que el Programa de Evaluación Docente en la UADY comenzó su implementación recientemente, resulta necesario documentar sus primeros resultados y compartir la experiencia con el fin de identificar aspectos de mejora que contribuyan al éxito del mismo. De la misma forma, se busca desarrollar una evidencia de su construcción y un punto de partida para futuros estudios relacionados.

\section{Metodología}

Tipo de estudio y diseño. Se presenta un estudio descriptivo con un enfoque mixto de investigación, predominantemente cuantitativo (Hesse-Biber, 2010). 
Participantes. Se presenta la información recopilada en los dos primeros semestres de la implementación del programa de evaluación. Estos son: agosto-diciembre de 2013 y enero-mayo de 2014. Los participantes son estudiantes y profesores del primer y segundo semestre de siete programas en los Campus de Ciencias de la Salud CCS (Facultad de Odontología, Química y Enfermería), Campus de Ciencias Exactas e Ingenierías CCEI (Facultad de Matemáticas) y el Campus de Ciencias Biológicas y Agropecuarias CCBA (Facultad de Veterinaria). El desglose se presenta en la tabla 3.

Tabla 3. Relación de profesores y estudiantes que participaron en el proceso de evaluación

\begin{tabular}{llcccc}
\hline CAMPUS & FACUltad & $\begin{array}{c}\text { ProfESORES } \\
\text { SEMESTRE } \\
\text { AGO-DIC 2013 }\end{array}$ & $\begin{array}{c}\text { ESTUDIANTES } \\
\text { SEMESTRE } \\
\text { AGO-DIC 2013 }\end{array}$ & $\begin{array}{c}\text { PrOFESORES } \\
\text { SEMESTRE } \\
\text { ENE-MAY } \\
\mathbf{2 0 1 4}\end{array}$ & $\begin{array}{c}\text { ESTUDIANTES } \\
\text { SEMESTRE } \\
\text { ENE-MAY } \\
\text { 2014 }\end{array}$ \\
\hline CCBA & Veterinaria & 17 & 156 & 20 & 80 \\
& Enfermería & 11 & 78 & 14 & 125 \\
CCS & Odontología & 10 & 83 & 12 & 94 \\
& Química & 14 & 102 & 16 & 88 \\
CCEI & Matemáticas & 9 & 91 & 8 & 59 \\
\hline Total & & 61 & 510 & 70 & 446 \\
\hline Furnter & & & & &
\end{tabular}

Fuente: Elaboración propia.

\subsection{Instrumentos}

Para recolectar la información sobre la evaluación se construyeron y validaron dos: el Instrumento de evaluación del profesor por parte de los estudiantes y el Instrumento de autoevaluación del profesor. Además, se solicitó la elaboración de un portafolio de evidencias. Se consideraron estos tres elementos como un primer acercamiento, dado que era necesario conocer la opinión del estudiante como usuario y poder contrastarla con la propia opinión del profesor. Por ello, se realizaron pruebas espejo de estudiantes y profesores. Por último, se consideró la elaboración de un portafolio de evidencias que sirva como un instrumento adicional de autoevaluación y reflexión del propio desempeño docente. Cada elemento se describe a continuación.

\subsubsection{Instrumento de evaluación del profesor por parte de los estudiantes}

Se compone de 35 ítems organizados en siete secciones (tabla 4). Para obtener el coeficiente de confiablidad se utilizó el Alpha de Cronbach, del cual se obtuvo un coeficiente total de 0,905. Esta puntuación se validó con la prueba de T de Hotelling con puntaje de 654,874 y p $=0,000$. En la tabla 4 se muestran los coeficientes obtenidos para cada sección. Cabe señalar que la última, denominada "satisfacción del estudiante”, no entró en el análisis por utilizar otra escala de respuesta.

Tabla 4. Relación de secciones e ítems correspondientes con su coeficiente de confiablidad

\begin{tabular}{lcc}
\hline \multicolumn{1}{c}{ SECCIONES } & ÍTEMS & $\begin{array}{c}\text { COEFICIENTE DE } \\
\text { CONFIABILIDAD }\end{array}$ \\
\hline Actividades como facilitador & $1-10$ & 0,75 \\
Actividades como evaluador & $11-18$ & 0,74 \\
Actividades como asesor & $19-24$ & 0,82 \\
Actividades como gestor & 25 & 0,74 \\
Actividades como tutor & $26-27$ & 0,73 \\
Actitud del profesor & $28-30$ & 0,81 \\
Satisfacción del estudiante & $31-35$ & \\
\hline
\end{tabular}

Fuente: Elaboración propia. 
Para las seis primeras secciones, en el período agosto-diciembre de 2013, se utilizó una escala de uno a cinco puntos, donde: $1=$ casi nunca, $2=$ ocasionalmente , $3=$ algunas veces, $4=$ frecuentemente y $5=$ casi siempre.

Posterior a la primera aplicación formal del instrumento, se realizó una metaevaluación, en la que se les pidió a los profesores que expresaran su opinión respecto a los instrumentos utilizados. El resultado de esta derivó en la adecuación de la escala utilizada, dado que se sugirió prescindir del punto medio y con ello evitar la tendencia a la respuesta neutral o indiferente. Por tanto, en el período de enero-mayo de 2014, se modificó de uno a cuatro puntos donde: $1=$ casi nunca, $2=$ algunas veces, 3 $=$ frecuentemente $\mathrm{y} 4=$ casi siempre.

En la última sección de la escala, se utilizó una escala de uno a tres puntos, donde $1=$ insatisfecho, $2=$ satisfecho y $3=$ muy satisfecho.

Por último, se incluyen cuatro preguntas abiertas en las que se consideran los aspectos beneficiosos de la asignatura, las fortalezas y áreas de mejora de los profesores y comentarios adicionales.

\subsubsection{Instrumento de autoevaluación del profesor}

Se compone de 36 ítems organizados en seis secciones (tabla 5).

Tabla 5. Relación de secciones e ítems correspondientes al instrumento de autoevaluación con su coeficiente de confiabilidad

\begin{tabular}{lcc}
\hline \multicolumn{1}{c}{ SECCIONES } & ÍTEMS & $\begin{array}{c}\text { COEFICIENTE DE } \\
\text { CONFIABLIDAD }\end{array}$ \\
\hline Actividades como facilitador & $1-10$ & 0,83 \\
Actividades como evaluador & $11-21$ & 0,83 \\
Actividades como asesor & $22-27$ & 0,87 \\
Actividades como gestor & $28-30$ & 0,82 \\
Actividades como tutor & $31-33$ & 0,79 \\
Actitud del profesor & $34-36$ & 0,88 \\
\hline
\end{tabular}

Fuente: Elaboración propia.

Utilizando el mismo procedimiento que para el instrumento de estudiantes, para obtener el coeficiente de confiabilidad se utilizó el Alpha de Cronbach, del cual se obtuvo un coeficiente total de 0,957. Esta puntuación se validó con la prueba de T de Hotelling con puntaje de 456,191 y p $=0$,000. En la tabla 5 se muestran los coeficientes obtenidos para cada sección.

De igual forma, en el período de agosto-diciembre de 2013, para estas seis secciones se utilizó una escala de uno a cinco puntos, donde: $1=$ casi nunca, $2=$ ocasionalmente, $3=$ algunas veces, $4=$ frecuentemente y $5=$ casi siempre.

Dado que el resultado de la metaevaluación de la primera aplicación formal del instrumento derivó en un cambio de escala para el instrumento de estudiantes y con la intención de mantener la evaluación espejo, en el período de enero-mayo de 2014 se utilizó una escala de uno a cuatro puntos, donde: $1=$ casi nunca, $2=$ algunas veces, $3=$ frecuentemente, $4=$ casi siempre.

Por último, se anexan tres preguntas abiertas en donde se consideran las fortalezas y áreas de mejora de los profesores y comentarios adicionales. 


\subsubsection{Portafolio de evidencias}

El portafolio consta de tres partes: introducción, evidencias y reflexión final.

a) La introducción se redacta en forma de ensayo, se solicita que se presenten los datos de identificación del profesor y la asignatura a evaluar y, además, se integra la filosofía de la docencia del profesor.

b) En el apartado de evidencias se anexan materiales y actividades utilizados dentro el proceso de enseñanza y aprendizaje como su planeación didáctica, evaluaciones y actividades de aprendizaje de los estudiantes, en donde se analice la pertinencia de dichas actividades, los criterios de evaluación utilizados para estas, así como las ventajas y desventajas del uso de las actividades en cuestión.

c) La reflexión final también se redacta a manera de ensayo y se analiza si se alcanzaron las competencias establecidas en la planeación didáctica, las estrategias y factores que favorecieron el desarrollo de las competencias, así como las que obstaculizaron, los cambios propuestos para futuros cursos, además de necesidades de capacitación o áreas de oportunidad en las que se compromete a trabajar para mejorar su desempeño docente.

Procedimiento: La recogida de información se realizó durante dos períodos escolares: agosto-diciembre de 2013 y enero-mayo de 2014. La evaluación se realizó con los profesores con asignaturas alineadas al MEFI y solo participaron las cinco facultades mencionadas con anterioridad. Para realizar la evaluación, al inicio de cada período escolar se solicitó en las facultades la relación de profesores y asignaturas a impartir y se establecieron fechas para la implementación de la evaluación previo al término de cada período escolar. Una vez obtenida la información, se elaboró una base de datos en SPSS para realizar el análisis cuantitativo de los cuestionarios para estudiantes y profesores. Aunado a esto, se realizó la categorización de las respuestas de las preguntas abiertas de cada cuestionario para agregarlas a los reportes de evaluación. Por último, se evaluó el portafolio del profesor por medio de una rúbrica.

Una vez conjuntada la información de cada elemento de evaluación, se generó un reporte por cada asignatura que impartió un profesor. Estos reportes se entregaron a los directivos de las facultades para trabajar la retroalimentación de los profesores.

\section{Resultados}

Para atender al objetivo de este trabajo, que es presentar la experiencia de su implementación y los resultados obtenidos en el primer año de ejecución, se muestran los resultados más relevantes obtenidos en el ciclo 2013-2014. La información se expone a modo de comparación por período escolar con: a) resultados generales, b) resultados generales por campus, c) resultados generales por facultad, d) comparaciones entre las respuestas de estudiantes y profesores por rol, e) análisis de comentarios de los estudiantes y profesores y, por último, f) resultados recabados en el portafolio del profesor.

\subsection{Resultados generales por semestre}

Es favorable observar que para el período de enero-mayo de 2014 se incrementó el porcentaje de profesores evaluados con nivel excelente y satisfactorio y no hubo profesores evaluados con el nivel necesita mejorar (gráfico 1). 


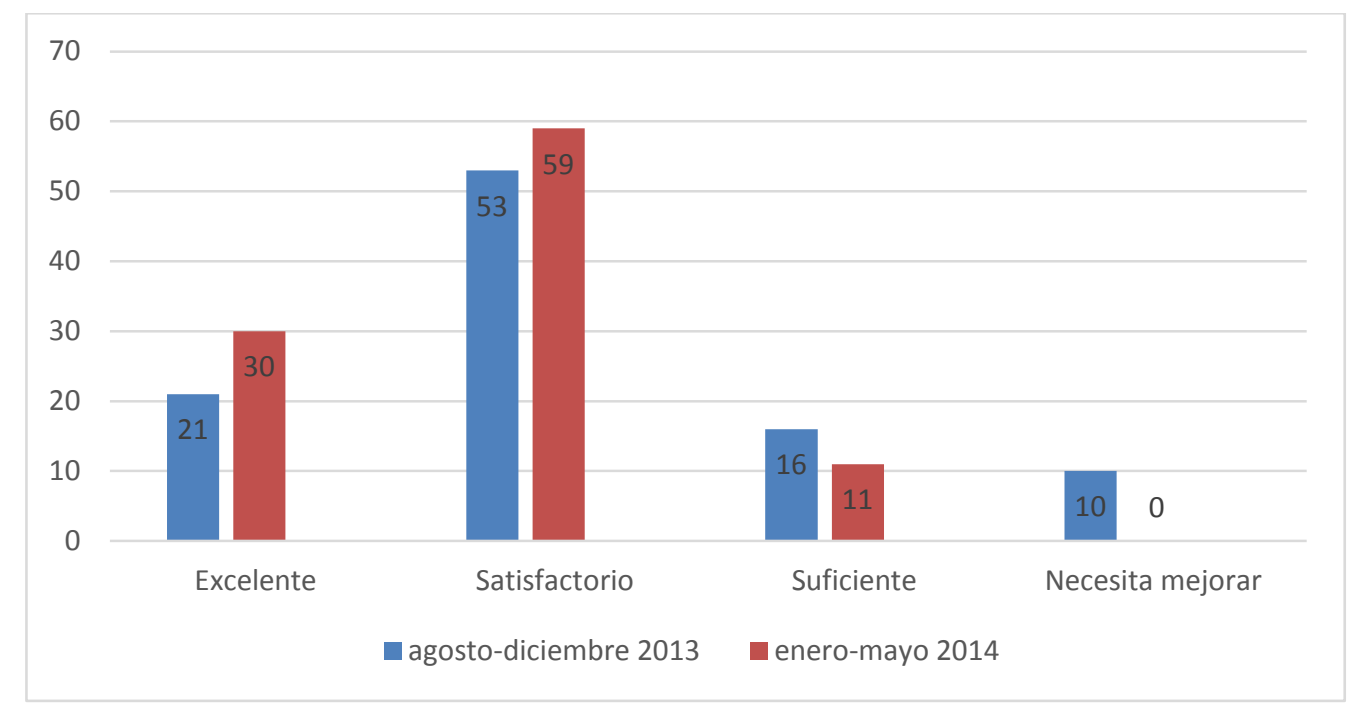

Gráfico 1. Comparación del porcentaje de profesores evaluados de acuerdo a su nivel de desempeño

Fuente: Elaboración propia.

\subsection{Resultados por campus: Comparación semestral}

Como se observa en la tabla 6, tanto en el Campus de Ciencias de la Salud, como en el de Ciencias Biológicas y Agropecuarias, se incrementó el porcentaje de profesores con nivel de desempeño excelente.

Tabla 6. Comparación del porcentaje de profesores evaluados de acuerdo a su nivel de desempeño por Campus y período de evaluación

\begin{tabular}{lcccccccc}
\hline $\begin{array}{c}\text { Nivel DE DESEMPEÑO } \\
\text { POR CAMPUS }\end{array}$ & \multicolumn{2}{c}{ EXCELENTE } & \multicolumn{2}{c}{ SATISFACTORIO } & SUFICIENTE & \multicolumn{2}{c}{$\begin{array}{c}\text { NECESITA } \\
\text { MEJORAR }\end{array}$} \\
\hline Período* & 1 & 2 & 1 & 2 & 1 & 2 & 1 & 2 \\
CCS & 20 & 36 & 42 & 49 & 25 & 15 & 11 & 0 \\
CCEI & 33 & 0 & 67 & 100 & 0 & 0 & 0 & 0 \\
CCBA & 17 & 28 & 65 & 62 & 6 & 10 & 12 & 0 \\
\hline
\end{tabular}

* Períodos: 1=agosto-diciembre 2014, 2=enero-mayo 2015

Fuente: Elaboración propia.

\subsection{Resultados por facultad: Comparacion semestral}

Específicamente, en las facultades donde se observó un incremento en el porcentaje de profesores con desempeño excelente fueron las de Química, Odontología y Veterinaria (tabla 7 ).

Tabla 7. Comparación del porcentaje de profesores de acuerdo a su nivel de desempeño por facultad y período de evaluación

\begin{tabular}{|c|c|c|c|c|c|c|c|c|}
\hline \multirow{2}{*}{$\begin{array}{c}\text { NIVEL DE } \\
\text { DESEMPEÑO POR } \\
\text { FACULTAD } \\
\text { Período* }\end{array}$} & \multicolumn{2}{|c|}{ EXCELENTE } & \multicolumn{2}{|c|}{ SATISFACTORIO } & \multicolumn{2}{|c|}{ SUfICIENTE } & \multicolumn{2}{|c|}{$\begin{array}{l}\text { NECESITA } \\
\text { MEJORAR }\end{array}$} \\
\hline & 1 & 2 & 1 & 2 & 1 & 2 & 1 & 2 \\
\hline Química & 7 & 13 & 43 & 62 & 29 & 25 & 21 & $\mathrm{O}$ \\
\hline Odontología & 10 & 58 & 40 & 42 & 10 & $\mathrm{O}$ & 10 & $\mathrm{O}$ \\
\hline Enfermería & 45 & 43 & 45 & 43 & 10 & 14 & 0 & $\mathrm{O}$ \\
\hline Matemáticas & 33 & 0 & 67 & 100 & $\mathrm{O}$ & $\mathrm{O}$ & 0 & $\mathrm{O}$ \\
\hline Veterinaria & 17 & 30 & 65 & 60 & 6 & 10 & 12 & $\mathrm{O}$ \\
\hline
\end{tabular}

* Períodos: 1 = agosto-diciembre 2014, 2 = enero-mayo 2015

Fuente: Elaboración propia. 
4.4. Comparación de las respuestas de estudiantes y profesores de acuerdo al rol: Período agosto-diciembre 2013

Como se observa en la gráfica 2, existe correspondencia entre la evaluación de estudiantes y profesores. El rol que salió menor puntuado en el período de agostodiciembre de 2013 fue el de gestor, mientras que el aspecto mejor evaluado es la actitud de los profesores.

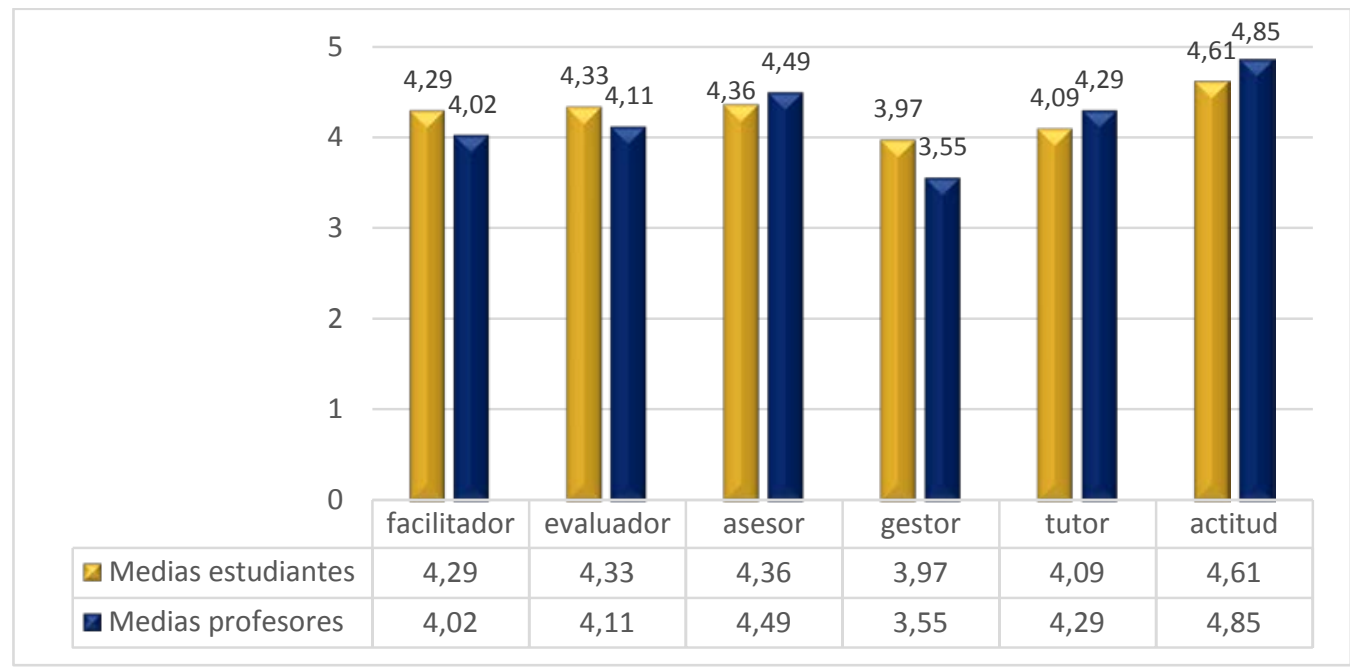

Gráfica 2. Comparación de estudiantes y profesores: Período agosto-diciembre 2013 Fuente: Elaboración propia.

Nuevamente, en el período de enero-mayo de 2014 se encontró correspondencia entre las puntuaciones otorgadas por cada rol. En este caso, los profesores se autoevaluaron más bajo en el rol de gestor y tanto estudiantes como profesores evaluaron más alto el aspecto de la actitud.

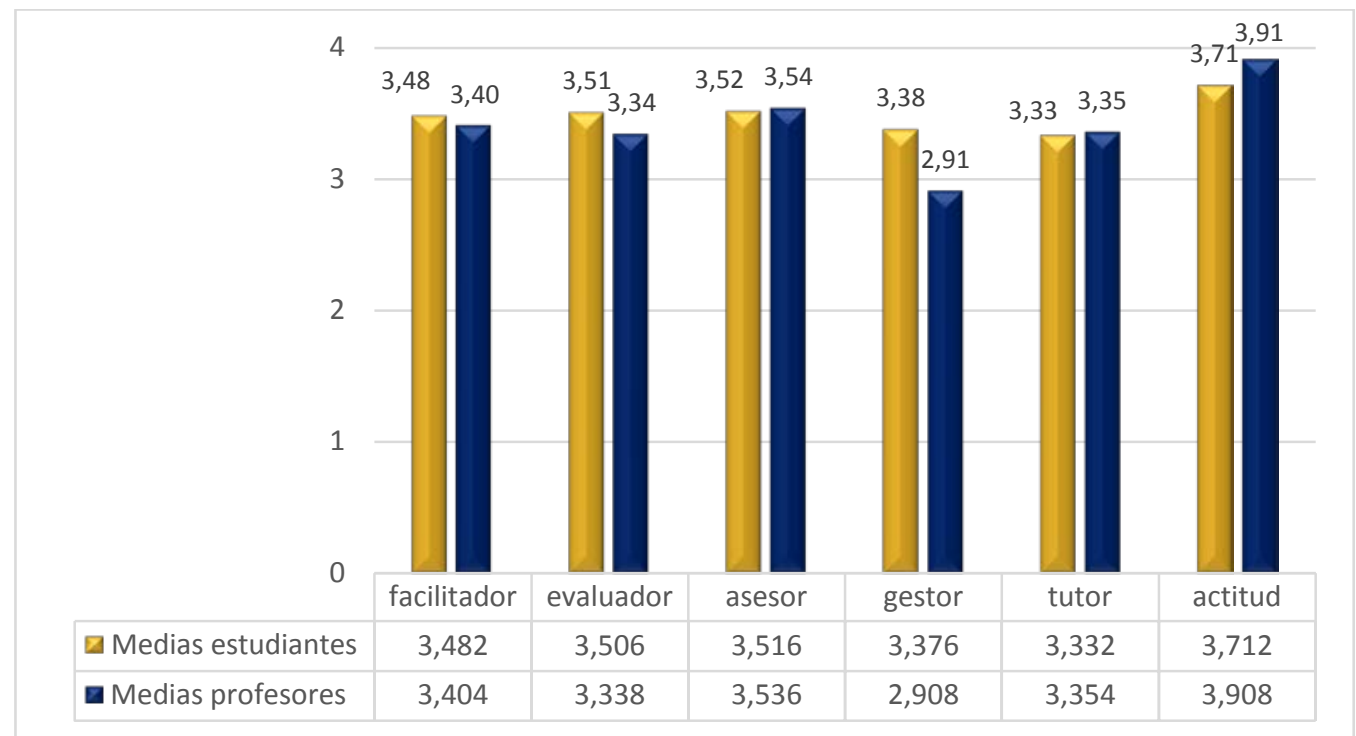

Gráfica 3. Comparación de estudiantes y profesores: Período enero-mayo 2014 Fuente: Elaboración propia. 


\subsection{Análisis de comentarios de los estudiantes y profesores}

Se categorizaron las respuestas a las preguntas abiertas del instrumento para estudiantes y profesores. Posteriormente, se agruparon de acuerdo a los que tuvieron mayor frecuencia en ambos períodos, agosto-diciembre de 2013 y enero-mayo de 2014.

Respecto a las fortalezas del profesor, las categorías más sobresalientes resultaron las siguientes:

a) Interés en el estudiante. Esta categoría agrupa los comentarios que hacen referencia a la preocupación del profesor porque el estudiante comprenda los temas y actividades. También hace referencia al diálogo que promueve el profesor, así como consejos para mejorar el desempeño en la asignatura. Algunos comentarios representativos son:

Se interesa en los estudiantes y puedes hablar con ella cuando quieras, está interesada en que aprendamos bien.

Es atento con los estudiantes, es buena persona y sabes que puedes hablar con él. Siempre tiene tiempo para nosotros.

Dialoga con el grupo y nos da consejos para un mayor desempeño y aprovechamiento de la materia.

b) Dominio del tema. Esta categoría agrupa los comentarios que hacen referencia a la explicación de los contenidos, la ejemplificación con situaciones reales y la experiencia de los profesores en el tema. Algunos comentarios representativos:

Tiene dominio y habilidad para poder explicar bien el tema.

Presentación clara y desarrollo efectivo de las competencias.

Conoce muy bien sus temas y es muy bueno explicándolos, apoya en horas extras si tienes dudas, era muy atento a todos y es divertido.

Cabe mencionar que esta fue una categoría que se comparte entre los comentarios de estudiantes y profesores; en el caso de los profesores algunos comentarios representativos:

Actualización en la asignatura que imparto y la relación de mis temas con los de otras asignaturas.

Capacidad para relacionar los contenidos con las competencias y el desarrollo de habilidades para la vida profesional.

Experiencia y conocimiento para crear escenarios reales de aprendizaje.

c) Habilidad para resolver dudas y problemas. Esta categoría agrupa los comentarios hechos por los estudiantes donde hacen referencia a la habilidad de los profesores para resolver dudas, tanto dentro como fuera del aula y por vías presenciales y no presenciales. Algunos comentarios representativos:

Resolvió dudas, lo que no entendías te lo volvía a explicar.

Siempre buscaba la manera de que entendamos mejor las cosas, dando asesorías con ayuda de otros profesores, en las salas de cómputo.

Siempre está disponible para ayudarnos a aclarar dudas dándonos asesoría incluso en horas que no son las de la clase.

d) Actitudes y habilidades. Esta categoría agrupa los comentarios que hacen referencia a las conductas, actitudes y habilidades que los estudiantes perciben como son la motivación, la puntualidad, la equidad en el trato y el manejo del grupo. Algunos comentarios representativos: 
Su paciencia y buen carácter nos fueron de mucha ayuda para poder avanzar en el estudio y comprensión en la materia.

Evalúa de manera justa los trabajos, en general es muy buen docente.

Es un profesor muy organizado, tiene la plataforma en buen estado.

Es un maestro atento y accesible, es excelente persona.

Demuestra gusto por la materia, se veía en que sus clases eran muy interesantes, eran didácticas y resolvía tus dudas.

Tiene carisma y es comprensivo.

En el caso de esta categoría, existe correspondencia en la opinión proporcionada por los profesores, algunos de los comentarios son:

Paciencia, tolerancia, disposición al diálogo y flexibilidad.

Motivar a los estudiantes para incorporarse a actividades fuera de la universidad para complementar su formación.

Empatía hacia los estudiantes, interés porque aprendan y actitud de respeto.

e) Estrategias y actividades de enseñanza y aprendizaje. Esta categoría agrupa los comentarios que hacen referencia a los aspectos positivos en cuanto al manejo de estrategias de enseñanza y aprendizaje como son el uso de actividades interesantes y significativas, incluir a la planeación didáctica más prácticas y salidas de campo, así como considerar actividades tanto individuales como grupales. Cabe señalar que esta categoría fue más representativa para profesores que para estudiantes. Algunos comentarios representativos de los profesores son:

Las actividades de aprendizaje variadas a las que se enfrentaron los estudiantes como prácticas de laboratorio y de campo.

El empleo de estrategias de enseñanza y aprendizaje significativos.

Gestionar actividades de aprendizaje en la asignatura y garantizar que las clases se cumplieron en tiempo y forma.

Sobre las áreas de mejora, se generaron las siguientes categorías:

a) Explicación de los temas y resolución de dudas. Agrupa los comentarios hechos por los estudiantes donde hacen referencia a los aspectos que puede mejorar el profesor en sus explicaciones. Algunos de los aspectos mencionados son el uso de términos no conocidos, ampliar las aportaciones que los estudiantes realizan en las exposiciones y desarrollar ejemplos durante las mismas. Algunas sugerencias son:

Que el lenguaje que utiliza en cada clase sea un poco más fácil de entender ya que en ocasiones se le olvida que somos iniciados en la carrera.

Que explique el tema directo y no le dé muchas vueltas y se vaya por otro tema.

Que no sólo se pare a hablar y explicar de un Word con el proyector, sino que al mismo tiempo que explique resuelva el ejercicio con una voz más potente.

Explicar más los procedimientos para realizar los problemas matemáticos, los ejercicios y tareas a realizar.

b) Estrategias y actividades de enseñanza y aprendizaje. Esta categoría agrupa los comentarios que hacen referencia a los aspectos que el profesor puede mejorar en cuanto al manejo de estrategias de enseñanza y aprendizaje como son el uso de actividades más dinámicas, proyección de videos, incluir a la planeación didáctica 
más prácticas y salidas de campo, así como considerar actividades tanto individuales como grupales. Algunos comentarios representativos son:

No dejar tantas ADAS cuando no hay sesiones presenciales.

Que relacione los temas vistos en clase con nuestro futuro medio laboral.

Que use videos, prácticas en el laboratorio y ejemplos en la vida cotidiana.

Que busque nuevas formas para aplicar los temas, no solo exponer el tema.

Una mejor distribución de ADAS durante todo el semestre.

Esta es una categoría compartida por estudiantes y profesores, al respecto los profesores comentaron:

Generé muchas actividades de aprendizaje.

Son demasiadas materias y demasiados trabajos que les marcamos los maestros.

Requiero capacitación en la búsqueda de nuevas estrategias de enseñanza.

c) Estrategias de evaluación. Esta categoría agrupa los comentarios que hacen referencia a los aspectos que puede mejorar en cuanto a la evaluación, como son aclarar el valor, criterios de evaluación y fechas de entrega de cada actividad, así como brindar retroalimentación de las mismas, no solo al final del semestre. Algunos comentarios son:

Aclarar el valor de las actividades y fechas para mantenernos al tanto.

Que dé retroalimentación de las actividades mucho antes, no hasta que acabe el semestre cuando ya registró la calificación.

Que se evalúe el conocimiento de los alumnos periódicamente para que el profesor esté atento y tenga conocimiento en lo que falta explicar.

Que cada vez que entre al salón, entre haciendo preguntas de lo que vieron en la clase anterior y con eso evaluar quién leyó.

Nuevamente, esta es una categoría compartida por la opinión de profesores y estudiantes. Al respecto los profesores comentaron:

Para mejorar las condiciones de enseñanza es necesario capacitarme en el diseño de instrumentos de evaluación.

d) Actitudes y habilidades. Esta categoría agrupa los comentarios relacionados con algunas acciones que se pueden mejorar como son el uso del celular en el salón de clase, la paciencia para responder y atender dudas, la atención a todos los estudiantes y no solo a un grupo particular, resaltar el valor y utilidad de cada asignatura, el manejo del grupo y cuidar la puntualidad y la asistencia a las sesiones presenciales. Algunos comentarios:

Que no sea tan dura, necesitamos más apoyo que llamadas de atención.

Que sea un poco más estricto.

Exigirles más a los alumnos y menos a él mismo, entiendo que él sabe mucho, pero debe dejar que hagamos algo o no aprenderemos.

Sugiero que debe tener más carácter al dirigirse a los alumnos, ya que en su mayoría, fueron muy groseros con los demás estudiantes en el aula y con él, pues no prestaban atención y hacían lo que querían, perjudicando a los que tratábamos de poner atención a la asignatura.

e) Manejo del tiempo. Esta categoría agrupa los comentarios hechos por los estudiantes donde hacen referencia a los aspectos que podrían considerarse en 
las planeaciones respecto a la distribución de los tiempos para el desarrollo de los temas y la resolución de actividades de aprendizaje.

La materia juntaba muchos conocimientos para un semestre. Debería haber otros semestres en los cuales se dé para que en verdad podamos aprender.

Esta asignatura requería de mayor tiempo en actividades presenciales, para despejar interrogantes y la participación de los estudiantes.

El tiempo que se otorga a esta asignatura es poco.

f) Uso de la plataforma. Esta categoría agrupa los comentarios que hacen referencia a los aspectos que podrían considerarse respecto al uso de la plataforma como un medio de interacción entre profesor y estudiantes, así mismo que se use como una herramienta didáctica para variar el estímulo y dinámica de las clases. Algunos comentarios:

Que mejore en el uso de la plataforma y la asignación de calificaciones.

El uso de la plataforma para poner recursos materiales que nos ayuden con los temas y no solo se use para subir tareas.

Que use un poco más la plataforma como apoyo para las actividades, utilizar más TICS en el planteamiento de los temas ya que la dinámica de la clase resultaba muy monótona porque se plantea con la pizarra únicamente.

Emplear y promover las TIC, mayor uso de la plataforma UADY Virtual.

Esta es una categoría que se comparte desde la perspectiva tanto de profesores como de estudiantes, algunos comentarios obtenidos en la autoevaluación son los siguientes:

Necesito más tiempo para optimizar la estructura y actividades plasmadas en la plataforma.

Para mejorar el aprendizaje se debería contar con equipo actualizado de computación y que la navegación de red sea rápida.

Requiero mayor capacitación en el uso de la plataforma.

\subsubsection{Resultados recabados con el portafolio de evidencias del profesor}

De acuerdo a las declaraciones realizadas en el apartado de reflexión, se obtuvieron los siguientes resultados:

a) Compromisos. Los profesores se comprometen a:

$\checkmark$ Asistir a cursos de capacitación en el área disciplinar.

$\checkmark$ Perfeccionar sus actividades de aprendizaje para que sean más integradoras.

$\checkmark$ Capacitarse en el uso de Tecnologías de la Información y Comunicación, particularmente en el uso de la plataforma UADY VIRTUAL.

$\checkmark$ Capacitación en la implementación del MEFI.

$\checkmark$ Actualización en el uso de estrategias de enseñanza y aprendizaje.

$\checkmark$ Entrega oportuna de la retroalimentación de las actividades de aprendizaje y uso de rúbricas más específicas y acordes con las actividades.

$\checkmark$ Reflexionar sobre los posibles errores que pudieron haberse cometido durante el curso y replicar las acciones que resultaron positivas para el desarrollo de las competencias planteadas en la asignatura.

$\checkmark \quad$ Fortalecer los roles establecidos en el MEFI.

$\checkmark$ Trabajar en equipo.

b) Áreas de mejora. Las áreas de mejora identificadas son: 
$\checkmark$ Capacitación en el uso de las TIC.

$\checkmark$ Capacitación en el uso de las estrategias de enseñanza y aprendizaje.

$\checkmark$ Reconsiderar la ponderación de las actividades.

$\checkmark$ Reestructurar las estrategias de evaluación.

$\checkmark$ Mejorar los instrumentos de evaluación.

$\checkmark$ Capacitación para desarrollarse mejor de acuerdo a los roles establecidos en el MEFI.

$\checkmark$ Incluir bibliografía en inglés.

c) Factores que dificultan el desempeño del profesor. La información proporcionada por el profesor se divide en factores internos y externos, tal como se presenta en la tabla 8 .

Tabla 8. Factores internos y externos que dificultan el desempeño del profesor

\begin{tabular}{|c|c|}
\hline INTERNOS & EXTERNOS \\
\hline $\begin{array}{l}\text { 1. Cantidad de estudiantes } \\
\text { por grupo. } \\
\text { 2. Diversidad de estudiantes } \\
\text { y sus conocimientos previos. } \\
\text { 3. Interés de los estudiantes. } \\
\text { 4. Estrategias de enseñanza } \\
\text { y aprendizaje utilizadas. } \\
\text { 5. Número excesivo de } \\
\text { actividades de aprendizaje. } \\
\text { 6. Elaboración de } \\
\text { instrumentos de evaluación } \\
\text { adecuados. }\end{array}$ & $\begin{array}{l}\text { 1.Inestabilidad de la plataforma UADY VIRTUAL. } \\
\text { 2. Fallas de internet e interrupciones en el servicio } \\
\text { eléctrico. } \\
\text { 3. Fallas en el equipo de cómputo. } \\
\text { 4. Programas extensos, en algunas asignaturas se } \\
\text { unieron los contenidos de dos materias del antiguo plan. } \\
\text { 5. Que el bachillerato no contribuye al desarrollo de } \\
\text { algunas competencias necesarias para ciertas } \\
\text { asignaturas. } \\
\text { 6. Dificultades familiares y económicas a las cuales se } \\
\text { enfrentan los estudiantes e influyen en su desempeño } \\
\text { académico. } \\
\text { 7. Las actividades de habilitación del MEFI interfirieron } \\
\text { con el tiempo destinado a su labor docente. }\end{array}$ \\
\hline
\end{tabular}

Fuente: Elaboración propia

\section{Conclusión y discusión}

Dentro de las principales aportaciones que se pudieron encontrar al presentar la descripción de la implementación de la evaluación, es favorable observar que incrementó el porcentaje de profesores evaluados con nivel satisfactorio y excelente, elevando de un $74 \%$ a un $89 \%$. Otro aspecto favorable es que, para el segundo período en que se realizó la evaluación, no hubo profesores ubicados en el nivel necesita mejorar. Esto puede reflejar la utilidad de la evaluación para retroalimentar el trabajo del profesor, ya que le permitió ubicar sus fortalezas y áreas de mejora y hacer los ajustes necesarios en el segundo semestre, en lugar de ser tomado con un mero elemento para tomar decisiones, en ocasiones financieras exclusivamente.

Otro aspecto que es importante señalar es la correspondencia que se observa en las respuestas otorgadas tanto por estudiantes como por profesores al evaluar el desempeño en cada uno de los roles. Esto indica en primera instancia que la evaluación se está realizando conscientemente y se analizan por ambos actores. El rol que tanto profesores como estudiantes evaluaron más bajo en ambos períodos fue el de gestor, por tanto, surge la necesidad de acompañar a los profesores para esclarecer y proponer actividades que les permitan cumplir con este rol.

Por otra parte, un aspecto que tanto profesores como estudiantes evaluaron más alto en ambos períodos es la actitud, lo que puede indicar la buena disposición de los profesores para generar espacios de respeto, diálogo y promoción de los valores universitarios. 
Esto va a la par de los comentarios realizados por los estudiantes y profesores cuando mencionan que algunas de las fortalezas son sus actitudes positivas, así como el interés en el estudiante; es decir, los estudiantes perciben a sus profesores como personas preocupadas por su aprendizaje, a los cuales pueden acudir y estos los tratarán paciente y respetuosamente. Por su parte, los profesores coinciden con esta perspectiva al considerarse profesionales que permiten el diálogo, se interesan por sus estudiantes y generan un ambiente de respeto. Estos elementos a su vez se relacionan con el rol de tutor del profesor de acuerdo a lo establecido en el MEFI (UADY, 2012).

Otro punto en el que coinciden estudiantes y profesores es en la percepción de estos como expertos en los contenidos de sus asignaturas, en su preparación académica y, además, por su experiencia docente. Esto es acorde a lo estipulado en el Plan de Desarrollo Institucional (PDI, 2010), en donde se establece que todos los académicos de la universidad se caracterizan por su amplia experiencia y preparación profesional, lo que les permite desarrollar competencias en la impartición del Modelo Educativo de la Universidad.

Sin embargo, también se identificaron áreas de mejora en la calidad de las explicaciones y en un aspecto en el que coinciden estudiantes y profesores es la necesidad de variar el uso de estrategias y actividades para hacer más dinámico el proceso de enseñanza y aprendizaje. Estos aspectos se relacionan con el rol de facilitador y a partir de estos resultados se está generando un programa de actualización y capacitación que brinde herramientas a los profesores.

De nueva cuenta, profesores y estudiantes consideran que los docentes deben ser más precisos en el uso de sus estrategias de evaluación, definir claramente sus criterios y proporcionar retroalimentación. A su vez, se identificaron otras áreas de mejora como actitudes poco favorables, como la falta de paciencia, el trato equitativo hacia los estudiantes, mejorar las habilidades en el manejo del grupo, así como mejorar la puntualidad y la asistencia a las sesiones presenciales. La atención de estas áreas de mejora puede considerarse una oportunidad en el proceso de transformación institucional y en el marco de la responsabilidad social universitaria al favorecer la mejora del perfil del profesorado, fomentando la conformación de una planta académica con altos niveles de habilitación, de acuerdo a lo establecido en el Plan de Desarrollo Institucional 2014-2022 (PDI, 2014).

De esta información, se generan recomendaciones en general y en específico que se trabajan al interior de las facultades. Algunas de estas son: manejar mejor los tiempos para dar oportunidad de abarcar todos los temas establecidos en la planeación didáctica, integrar a esta actividades de aprendizaje diversas e inclusive utilizar la plataforma virtual como recurso de apoyo en el proceso de enseñanza y aprendizaje. Otro aspecto importante a considerar es brindar retroalimentación periódica de las actividades y evaluaciones realizadas con el fin de monitorear sus avances durante el semestre.

Es interesante apuntar que las áreas de mejora identificadas por los estudiantes y profesores también coinciden con las áreas mencionadas por los propios profesores en su portafolio, ya que estos solicitan capacitación en el uso de las tecnologías de información y comunicación (TIC), en especial de la plataforma virtual, así como en el diseño e implementación de estrategias de enseñanza-aprendizaje y evaluación. De modo que los profesores se comprometen a capacitarse en aspectos disciplinares, lo concerniente al Modelo Educativo, en el uso de las TIC. De igual forma, a continuar actualizándose el ámbito pedagógico para mejorar el diseño de sus planeaciones 
didácticas, el diseño de actividades de aprendizaje más integradoras y mejorar los instrumentos de evaluación utilizados en el proceso de enseñanza y aprendizaje.

Por último, es necesario reflexionar sobre los factores internos y externos que dificultan el desempeño del profesor. En el caso de los factores internos, se encuentran en primer plano el número de estudiantes, así como los conocimientos previos, características e intereses de estos. Dada la naturaleza pública de la universidad, sería poco viable la reducción de estudiantes aceptados, sin embargo, es posible la capacitación de los profesores para el manejo de grupos numerosos, la generación de estrategias acordes a las características de los grupos, así como la reducción de las actividades de aprendizaje. Es decir, cambiar la perspectiva de las "tareas" al diseño de experiencias de aprendizaje más integradoras con criterios de evaluación claramente establecidos, esto con el fin de facilitar el trabajo de los profesores en el curso de sus asignaturas.

Por otra parte, se encuentran los factores externos como son la inestabilidad de la red, lo que dificulta el uso de la plataforma virtual, aunado a las fallas del equipo de cómputo de las facultades. Además, al realizarse la reestructura de los planes de estudio, algunas asignaturas se fusionaron en una sola, por lo que los profesores perciben que los programas son más extensos y no cuentan con el tiempo necesario para abarcar los contenidos. Es importante trabajar en la capacitación de los profesores relativa al desarrollo de competencias, ya que continúan dando mayor importancia al contenido, el cual es solo un elemento de la competencia y no dimensiona el trabajo integral que implica el desarrollo de competencias.

\section{Referencias}

Aguilar, V. (2009, septiembre). Sistema de evaluación docente. Hacia un modelo formativo e integral en educación superior. Ponencia presentada en el X Congreso Nacional de Investigación Educativa, Veracruz.

Bonifaz, R. (2011). Origen de la evaluación docente y su conexión con las políticas públicas en educación. En J. Manzi, R. González y Y. Sun (Eds.), La evaluación docente en Chile (pp. 13-34). Ciudad de México: Centro de Medición MIDE.

Cisneros, E. y Aguilar, Z. (2010). Validación de un modelo de desarrollo y evaluación de competencias académicas en el sureste de México. En E. Cisneros, B. García, E. Luna y R. Marín (Coords.), Evaluación de competencias docentes en Educación Superior (pp. 243266). Ciodad de México: REDECA.

De Miguel, M. (1998). La evaluación del profesorado universitario. Criterios y propuestas para mejorar la función docente. Revista de Educación, 315, 67-83.

García, B., Loredo, J., Luna, E. y Rueda, M. (2008). Modelo de Evaluación de Competencias Docentes para la Educación Media y Superior. Revista Iberoamericana de Evaluación Educativa,1(3e), 124-136.

Rizo, H. (1999). Evaluación del docente universitario. Revista Electrónica Interuniversitaria del Formación del Profesorado, 2(1), 425-440.

Rueda, M. (2008). La evaluación del desempeño docente en la universidad. Revista Electrónica de Investigación Educativa, 10, 1-15.

Hesse-Biber, S. (2010). Mixed methods research: Merging theory with practice. Nueva York: Guilford Press. 
SEP (2010). Lineamientos de evaluación docente. Evaluación del desempeño docente bajo el enfoque por competencias. Ciudad de México: Dirección General de Bachillerato.

UADY. (2012). Modelo Educativo para la Formación Integral. Mérida (Yucatán): Autor. 